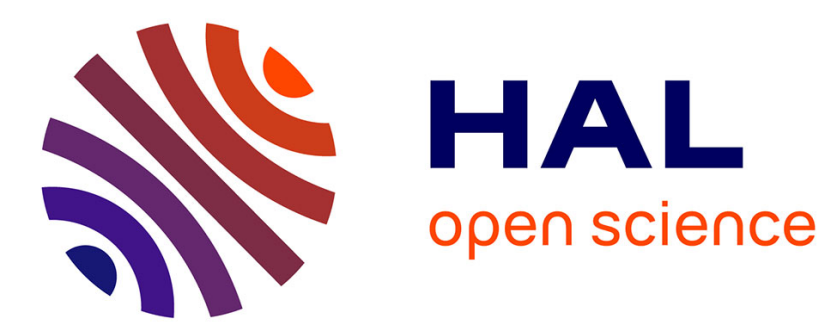

\title{
Étude expérimentale des effets opto-mécaniques sur fibres pour télécommunications optiques
}

\author{
A. Zaganiaris, F. Alard
}

\section{To cite this version:}

A. Zaganiaris, F. Alard. Étude expérimentale des effets opto-mécaniques sur fibres pour télécommunications optiques. Revue de Physique Appliquée, 1977, 12 (5), pp.805-807. 10.1051/rphysap:01977001205080500 . jpa-00244245

\section{HAL Id: jpa-00244245 https://hal.science/jpa-00244245}

Submitted on 1 Jan 1977

HAL is a multi-disciplinary open access archive for the deposit and dissemination of scientific research documents, whether they are published or not. The documents may come from teaching and research institutions in France or abroad, or from public or private research centers.
L'archive ouverte pluridisciplinaire HAL, est destinée au dépôt et à la diffusion de documents scientifiques de niveau recherche, publiés ou non, émanant des établissements d'enseignement et de recherche français ou étrangers, des laboratoires publics ou privés. 


\title{
ÉTUDE EXPÉRIMENTALE DES EFFETS OPTO-MÉCANIQUES SUR FIBRES POUR TÉLÉCOMMUNICATIONS OPTIQUES
}

\author{
A: ZAGANIARIS et F. ALARD \\ Centre National d'Etudes des Télécommunications \\ Département P. M. T., 22301 Lannion, France
}

\begin{abstract}
Résumé. - Nous présentons des résultats expérimentaux sur la dégradation des caractéristiques optiques des fibres pour télécommunications lorsqu'elles sont soumises à une contrainte de tension. Après avoir localisé les défauts mécaniques à l'intérieur de la fibre, on enregistre leur évolution sous contrainte croissante. Des résultats de fatigue statique sont également présentés.
\end{abstract}

Abstract. - We report experimental evidence of the optical degradation of a fiber under tensile stress. The localization of the fiber defects, their evolution under increasing stress and static fatigue results are presented.

1. Introduction. - Les progrès technologiques réalisés dans le domaine des fibres optiques ont permis d'atteindre des niveaux d'atténuation extrêmement bas. L'intérêt des laboratoires concernés se déplace en partie vers une meilleure connaissance des caractéristiques mécaniques des fibres. En effet, celles-ci, pendant le processus du câblage et lors de l'utilisation de ce câble, devront résister à de multiples contraintes.

Les propriétés mécaniques des fibres ont été fort peu étudiées jusqu'à présent ; on a rapporté des mesures statistiques de la force de rupture en traction [1] en liaison avec le revêtement protecteur de la fibre. Dans cet article, on décrit une méthode pour la mise en évidence de la dégradation continue d'une fibre optique lorsqu'une contrainte graduellement croissante y est appliquée. Cette dégradation commence, en général, bien avant le point de rupture et doit être prise en considération lors de l'emploi des fibres sur le terrain où elles seront sous contrainte continue.

Cette méthode permet de visualiser les défauts à l'intérieur d'une fibre et sur sa surface et de suivre leur évolution en fonction de la contrainte appliquée. Les défauts présents dans le cœur et dans la gaine de la fibre sont séparés et on en tire des renseignements sur leur taille et sur leur nature.

Le vieillissement des fibres sous contrainte constante peut être continuellement enregistré jusqu'à la rupture.

2. Le montage expérimental. - Le dispositif comporte principalement :

Un laser avec un modulateur et l'optique d'injection de la lumière dans la fibre.

Un cube intégrateur [2] composé de 24 cellules solaires entourant la fibre et mobile le long de celle-ci. Ce cube intègre la lumière diffusée par les défauts

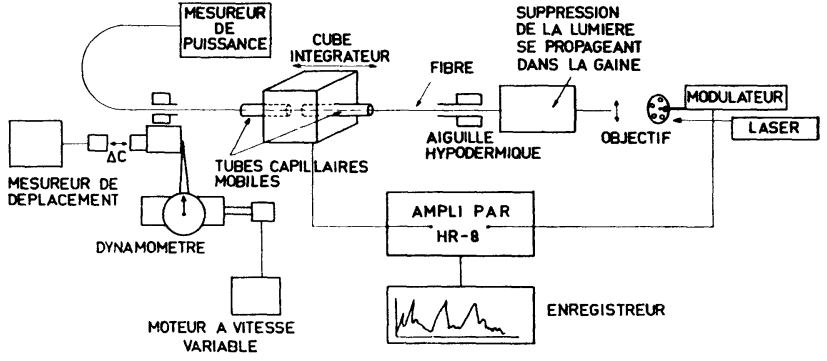

FIG. 1. - Le montage expérimental.

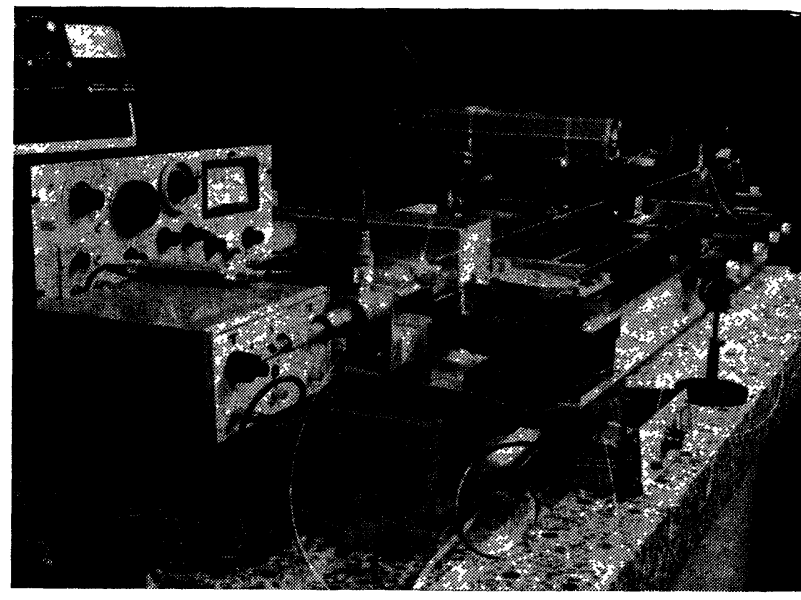

Fig. 2. - Vue d'ensemble.

présents dans une faible longueur de l'échantillon, de l'ordre de $1 \mathrm{~mm}$.

Un montage permettant d'appliquer une force de traction pure sur la fibre. Pour cela, celle-ci est collée à ses extrémités à l'intérieur de deux aiguilles hypodermiques qui. grâce à une fixation à 3 degrés de liberté, 
s'alignent automatiquement dès qu'une force est appliquée. L'échantillon est ainsi soumis à une traction axiale pure.

L'ensemble d'amplification et d'enregistrement du signal issu du cube intégrateur. Sont également enregistrés les signaux correspondant à la force appliquée et à la position du cube le long de la fibre. Ces derniers sont engendrés par des potentiomètres linéaires intégrés au montage.

Un mesureur capacitif de déplacement pour la mesure à distance de l'allongement de la fibre.

Eventuellement, une enceinte hygrométrique entourant l'échantillon.

Les fibres examinées jusqu'à présent étaient du type CVD ou CVR et provenaient du C.N.E.T. et de Corning Glass Works.

3. Localisation des défauts diffusants. - Les conditions de couplage choisies sont telles que la lumière du laser est injectée autant dans la gaine que dans le cœur de la fibre. La gaine étant en silice de bonne qualité, laisse la lumière se propager sur plusieurs mètres sans atténuation notable. De ce fait, à l'endroit de la mesure, la lumière diffusée provient des défauts présents dans l'ensemble de la fibre.

Si maintenant on supprime la lumière se propageant dans la gaine avant le point de mesure, l'enregistrement obtenu sera l'image des défauts présents uniquement dans le cœur de la fibre. La figure 3 est un exemple de ce procédé simple qui permet de localiser les centres diffusants dans la fibre.

La suppression de la lumière se propageant dans la gaine s'obtient soit par immersion de la fibre dans un liquide d'indice de réfraction supérieur à celui de la gaine, soit par la coloration noire de celle-ci.

L'examen de la figure 3 révèle les différents types de centres diffusants. Ceux du type 1 appartiennent au cour de la fibre tandis que ceux du type 3 à la gaine ; enfin, les défauts du type 2 semblent affecter le cœur et la gaine à la fois.

La photographie de la figure 3 a montré la surface de rupture survenue au point 2 ; l'hétérogénéité diffusante fut à l'origine de la fracture.

4. L'influence de la contrainte. - L'évolution des centres diffusants en fonction de la contrainte appliquée à la fibre est représentée par les enregistrements de la figure 4.

Nous avons constaté que cette évolution a un caractère aléatoire : un défaut important à l'origine peut rester inchangé ou évoluer très peu avec la contrainte tandis que de nouveaux défauts apparaissent et prennent de l'importance. Ceci n'est pas toujours le cas.

Pour les fibres que nous avons testées, la fracture se produit dans la majorité des cas grâce à un défaut de surface. En protégeant les fibres nues d'une mince pellicule plastique, la résistance à la traction peut jusqu'à doubler de valeur, mais l'origine des fractures

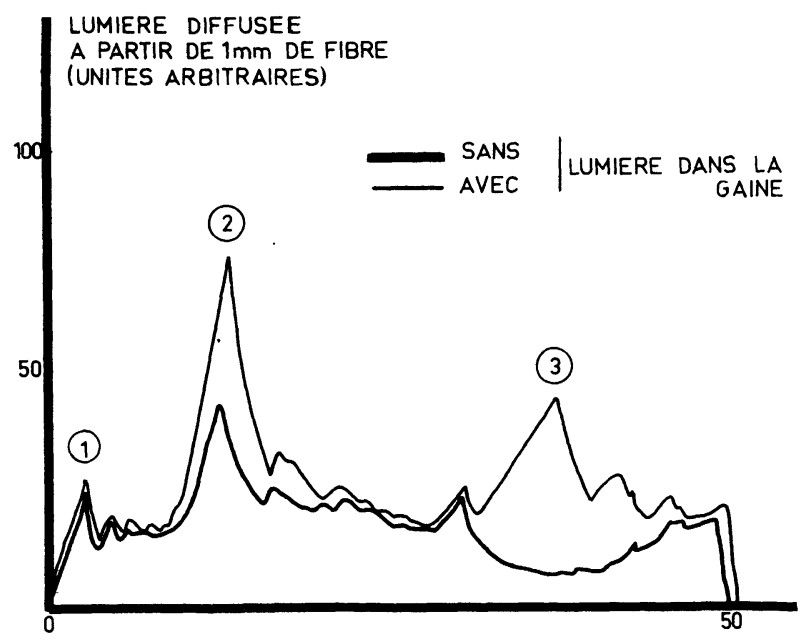

DISTANCE LE LONG DE LA FIBRE $(\mathrm{cm})$

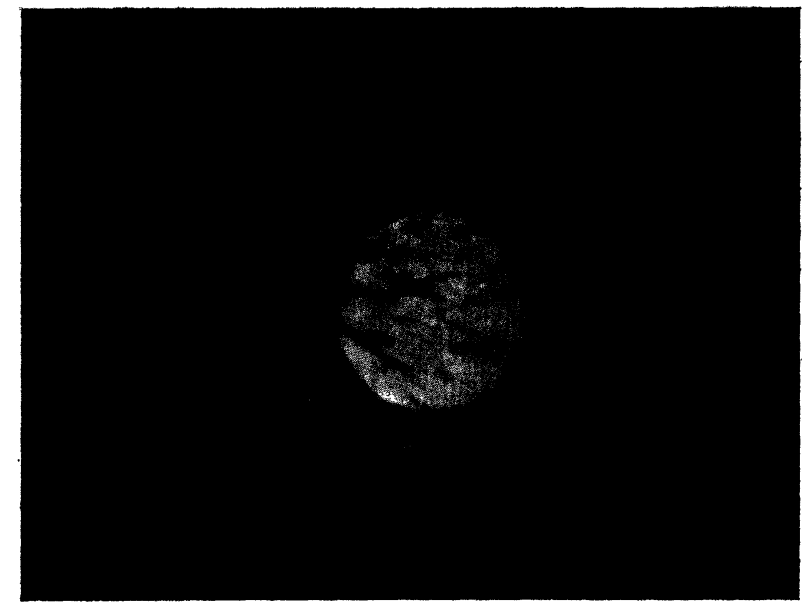

FIG. 3. - Localisation des centres diffusants dans une fibre optique.

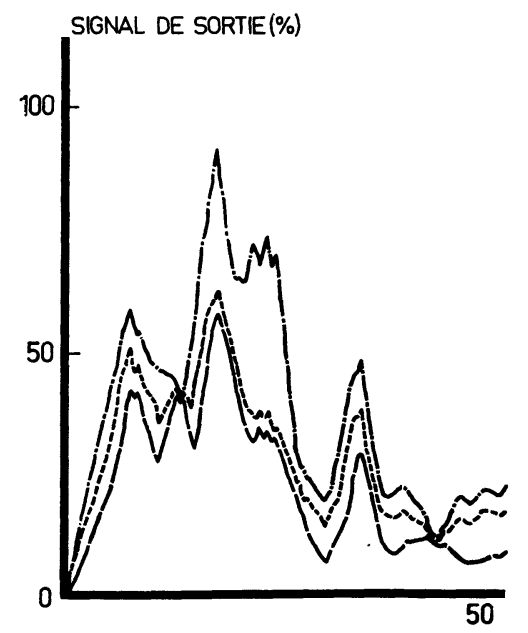

DISTANCE LE LONG DE LA FIBRE (cm)

FIG. 4. - L'influence de la contrainte sur les centres diffusants : - - Sans contrainte ; - - $100 \mathrm{~g}$; - - - $200 \mathrm{~g}$. 
semble rester liée à des amorces superficielles dans la plupart des cas, comme pour les fibres non protégées.

5. Fatigue statique des fibres. - La fatigue statique des fibres de verre textile a été étudiée extensivement [3] et pourrait en partie s'appliquer aux fibres optiques. En effet, dans les deux cas, le comportement des fibres sous charge constante est principalement influencé par :

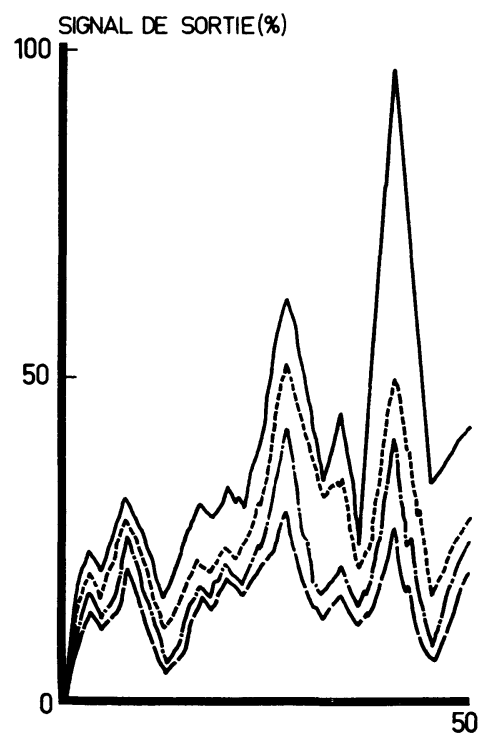

DISTANCE LE LONG DE LA FIBRE (cm)

Fig. 5. - Fatigue statique d'une fibre sous contrainte de tension constante : - - Enregistrement initial ; - - - - Après 10 heures ; - . . . . . Après 20 heures ; —— Après 60 heures.
- l'état de surface de la fibre : corrosion superficielle, adsorption de substances étrangères, importance des entailles;

- le type et la durée de la contrainte et

- l'atmosphère entourant l'échantillon pendant la mesure : température, humidité, environnement chimique.

La figure 5 résume les résultats obtenus sur une fibre C.N.E.T. protégée par une mince pellicule plastique, et dont nous avons voulu suivre le vieillissement sous charge de traction constante. Des expériences sont en cours sous atmosphère contrôlée.

6. Conclusion. - Nous venons de décrire nos premières expériences pour déterminer les effets des contraintes mécaniques sur les fibres optiques.

Comme contrainte, nous avons choisi la traction car c'est elle qui donne le plus de renseignements et qui s'approche le plus des conditions réelles. Il est envisagé d'entreprendre aussi des mesures sous torsion et sous contraintes combinées.

Nous avons mis en évidence une fragilisation importante des fibres même sous faible contrainte. Cette dégradation pourrait affecter la propagation de la lumière dans un câble souterrain à fibres optiques.

La visualisation des défauts, leur évolution lorsqu'une charge est appliquée et l'examen des surfaces de rupture devraient permettre de remonter à leur nature et taille et de lier ces caractéristiques aux conditions de fabrication des fibres.

\section{Bibliographie}

[1] France, P. W., Dunn, P. L., Protection des fibres optiques par une solution plastique, $2^{\mathrm{e}}$ Coll. Eur. Transm. Fibres Opt., Paris Sept. 1976.

[2] TyNeS, A. R. et Coll. J. O. S. A. 61 (1971) 143.

[3] Mould, R. E., Glass tech. Bericht III (1959) 18. 